\title{
Eigenstate thermalization hypothesis (ETH) and integrability in quantum spin chains
}

\author{
Vincenzo Alba ${ }^{1,2}$ \\ ${ }^{1}$ Department of Physics and Arnold Sommerfeld Center for Theoretical Physics, \\ Ludwig-Maximilians-Universität München, D-80333 München, Germany \\ ${ }^{2}$ International School for Advanced Studies (SISSA), Via Bonomea 265, 34136, Trieste, Italy, INFN, Sezione di Trieste
}

(Dated: April 22, 2015)

\begin{abstract}
We investigate the eigenstate thermalization hypothesis $(\mathrm{ETH})$ in integrable models, focusing on the spin- $\frac{1}{2}$ isotropic Heisenberg $(X X X)$ chain. We provide numerical evidence that ETH holds for typical eigenstates (weak ETH scenario). Specifically, using a numerical implementation of state-of-the-art Bethe ansatz results, we study the finite-size scaling of the eigenstate-to-eigenstate fluctuations of the reduced density matrix. We find that fluctuations are normally distributed, and their standard deviation decays in the thermodynamic limit as $L^{-1 / 2}$, with $L$ the size of the chain. This is in contrast with the exponential decay that is found in generic nonintegrable systems. Based on our results, it is natural to expect that this scenario holds in other integrable spin models and for typical local observables. Finally, we investigate the entanglement properties of the excited states of the $X X X$ chain. We numerically verify that typical mid-spectrum eigenstates exhibit extensive entanglement entropy (i.e., volume-law scaling).
\end{abstract}

\section{INTRODUCTION}

In classical physics the observation that at long times systems thermalize led to the birth of statistical mechanics as a very effective description of nature. However, the issue of how equilibration and thermalization arise in isolated quantum (many-body) systems is still highly debated [1-7]. Recent years have witnessed a resurgence of interest in this topic [2, 4, 7-52], due to its relevance in cold atoms experiments [53-66]. One possible mechanism explaining thermalization is the so-called eigenstate thermalization hypothesis (ETH) [1, 3, 4, 18, 67-84].

The ETH can be stated as follows: In the thermodynamic limit the eigenstate expectation value $(\mathrm{EEV}) \widehat{\mathcal{O}}_{\alpha \alpha} \equiv$ $\left\langle\psi_{\alpha}|\widehat{\mathcal{O}}| \psi_{\alpha}\right\rangle$ of a typical few-body observable $\widehat{\mathcal{O}}$ in an eigenstate $\left|\psi_{\alpha}\right\rangle$ of a many-body Hamiltonian $\mathcal{H}$ with eigenenergy $E_{\alpha}$ (and energy density $e_{\alpha} \equiv E_{\alpha} / L$, with $L$ being the number of sites) is equal to the microcanonical average $\langle\widehat{\mathcal{O}}\rangle$, at the mean energy density $\langle\mathcal{H} / L\rangle=e_{\alpha}$, i.e.,

$$
\widehat{\mathcal{O}}_{\alpha \alpha}=\langle\widehat{\mathcal{O}}\rangle, \quad \text { with }\langle\widehat{\mathcal{O}}\rangle \equiv \mathcal{N}^{-1}\left(e_{\alpha}, \Delta e\right) \sum_{\gamma:\left|e_{\gamma}-e_{\alpha}\right|<\Delta e} \widehat{\mathcal{O}}_{\gamma \gamma}
$$

Here $\Delta e$ is a small energy scale that can be sent to zero in the thermodynamic limit, and $\mathcal{N}\left(e_{\alpha}, \Delta e\right)$ denotes the number of energy levels $e_{\gamma}$ in the energy window $\left|e_{\alpha}-e_{\gamma}\right|<\Delta e$. The underlying idea of ETH is that eigenstates with similar energy give similar EEVs. As a consequence, the standard deviation of the fluctuations, $\sigma(\widehat{\mathcal{O}}) \equiv\left[\left\langle\widehat{\mathcal{O}}^{2}\right\rangle-\langle\widehat{\mathcal{O}}\rangle^{2}\right]^{1 / 2}$, vanishes in the thermodynamic limit, which implies that $\widehat{\mathcal{O}}_{\alpha \alpha}$ becomes a "smooth" function of $e_{\alpha}$.

Still, two main interpretations of ETH are possible [26]. In the so-called weak ETH, "rare" (as opposed to typical [3, 7173]) eigenstates $\left|\psi_{r}\right\rangle$, for which $\widehat{\mathcal{O}}_{r r} \neq\langle\widehat{\mathcal{O}}\rangle$, are allowed for any finite size. The behavior of the fluctuations reflects that the fraction of rare eigenstates vanishes in the thermodynamic limit. Interestingly, these rare eigenstates appear for Hamiltonians corresponding to random matrices [85]. On the other hand, in the strong ETH these rare eigenstates are not present, i.e., all eigenstates are thermal. This difference is dramatically reflected in the finite-size scaling behavior of $\sigma(\widehat{\mathcal{O}})$, and it is related to the difference between integrable [86] and non integrable models. In the latter the strong ETH holds, and it is now well established that fluctuations decay exponentially with system size $[4,27,38,76,77,79,80,83]$. Exact diagonalization studies demonstrated that for typical observables $\sigma(\widehat{\mathcal{O}}) \propto D^{-1 / 2}$ [77], with $D$ the dimension of the full Hilbert space. Oppositely, in integrable models a much slower decay is expected $[4,67]$, which is associated with the presence of an extensive number of local conservation laws [86]. Yet, despite integrability, numerical studies of ETH are hampered by severe finite-size effects, and a precise finite-size scaling analysis of $\sigma(\widehat{\mathcal{O}})$ is still lacking (see, however, Ref. 75 for a finite-size scaling study of ETH in the Lieb-Liniger model).

\section{SUMMARY OF THE RESULTS}

In this work, using a numerical implementation (cf. Ref. 87 for the details) of state-of-the-art Bethe ansatz results [88, 89], we perform a finite-size scaling analysis of the ETH in the spin- $\frac{1}{2}$ isotropic Heisenberg $(X X X)$ chain. Specifically, here we focus on the $\ell$-spin reduced density matrix $\rho_{\ell}$. Given the state of the chain $|\psi\rangle$ and a block $A$ of $\ell$ contiguous spins, the corresponding $\rho_{\ell}$ is obtained by tracing over the degrees of freedom of the remaining spins (block $B$ ) in the full-system density matrix $\rho \equiv|\psi\rangle\langle\psi|$, i.e., $\rho_{\ell} \equiv \operatorname{Tr}_{B} \rho$ [90]. From $\rho_{\ell}$ it is straightforward to obtain any multi-spin correlation function with support on $\ell$ contiguous sites of the chain.

Our main result is that the weak ETH holds for all the matrix elements of $\rho_{\ell}$, meaning that for the vast majority of the eigenstates (i.e., typical eigenstates) one has $\rho_{\ell} \rightarrow\left\langle\rho_{\ell}\right\rangle$ (with $\langle\cdot\rangle$ denoting the microcanonical average), in the thermodynamic limit. This scenario is made rigorous by a large scale analysis of the eigenstate-to-eigenstate fluctuations of $\rho_{\ell}$. Precisely, we numerically demonstrate that fluctuations are normally distributed, and their standard deviation $\sigma\left(\rho_{\ell}\right)$ decays as $\sigma\left(\rho_{\ell}\right) \propto L^{-1 / 2}$, for large chains. Interestingly, the same be- 

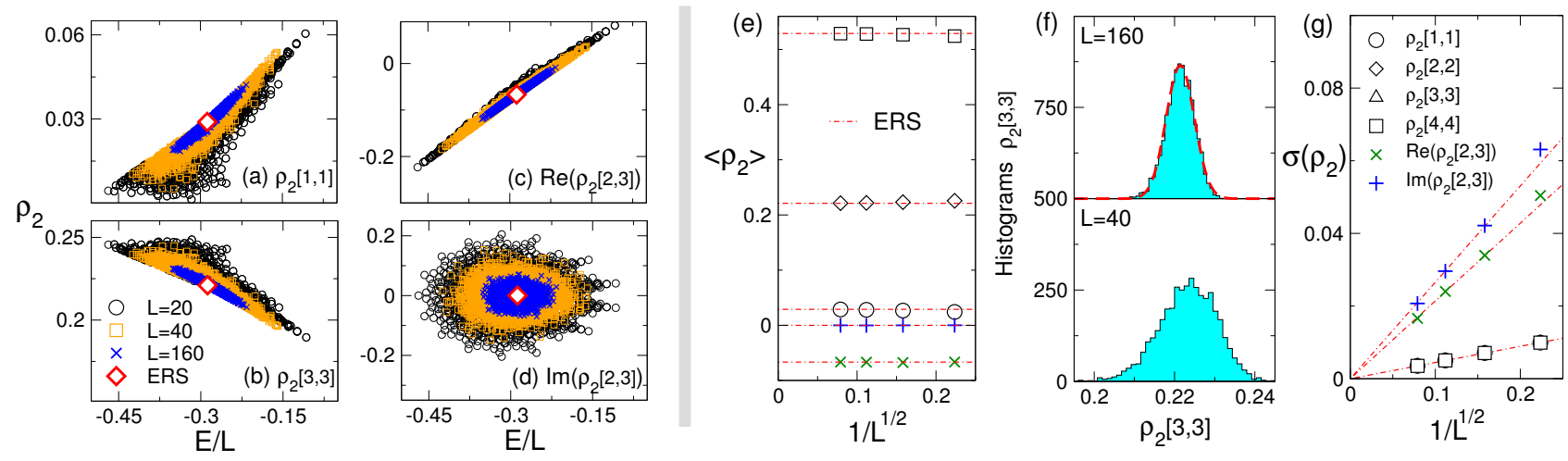

FIG. 1. Finite-size scaling of the ETH for the two-spins reduced matrix $\rho_{2}$ in the $X X X$ chain. (a)-(d) Matrix elements $\rho_{2}[1,1], \rho_{2}[3,3]$, and $\rho_{2}[2,3]$ plotted versus the eigenstates energy density $E / L$. Panels (c) and (d) plot the real and imaginary part of $\rho_{2}[2,3]$, respectively. Circles, squares, and crosses denote chain sizes $L=20,40,160$. The data correspond to $\sim 10^{4}$ eigenstates obtained from real solutions of the Bethe equations at fixed particle density $x \equiv M / L=1 / 4$, with $M$ the number of down spins (particles). In all panels the rhombi denote the result obtained from the ensemble representative state (ERS). All the matrix elements are well described by the ERS in the limit $L \rightarrow \infty$. (i) Eigenstate average $\left\langle\rho_{2}\right\rangle$ plotted versus $L^{-1 / 2}$. Different symbols correspond to different matrix elements (cf. (iii)). The dashed-dotted lines are the results obtained from the ERS. (ii) Eigenstate-to-eigenstate fluctuations of $\rho_{2}$. Histograms of $\rho_{2}[3,3]$ obtained from different eigenstates and $L=40,160$. The histogram for $L=160$ is shifted vertically for visibility. The dashed line is a gaussian fit. (iii) Fluctuations of $\rho_{2}$ : Standard deviation $\sigma\left(\rho_{2}\right)$ plotted versus $L^{-1 / 2}$. The dashed-dotted lines are linear fits. $\sigma\left(\rho_{2}\right)$ is vanishing in the limit $L \rightarrow \infty$.

havior is observed in free models [26], and it is in sharp contrast with the exponential decay found in non-integrable systems [77]. Moreover, using standard thermodynamics Bethe ansatz (TBA) results [91-93], we provide an ensemble representative state (ERS), and the reduced density matrix $\rho_{\ell}^{E R S}$ thereof. This is a good approximation for the reduced density matrix obtained from typical mid-spectrum eigenstates of the finite chain. In particular, one has $\rho_{\ell} \rightarrow \rho_{\ell}^{E R S}$ in the thermodynamic limit. From $\rho_{\ell}$ we construct the conserved charges $I_{n}(n \in \mathbb{N})$ of the $X X X$ chain [94]. Interestingly, we numerically observe that the EEV of the first non-trivial charge densities $I_{n} / L$ vanishes for typical eigenstates. One consequence of our results is that, despite the $X X X$ chain being integrable, the unitary dynamics ensuing from thermallike initial states might lead to thermal behavior at long times, in agreeement with what has been found in Ref. 95 and 96. Finally, we investigate the entanglement entropy [90, 97-99] $S_{\ell} \equiv \operatorname{Tr} \rho_{\ell} \log \rho_{\ell}$ of the ERS. We provide robust numerical evidence for the volume law scaling $S_{\ell}^{E R S} \propto \ell$, which implies that typical mid-spectrum eigenstates exhibit extensive entanglement entropy.

\section{THE MODEL AND THE METHOD}

The spin- $\frac{1}{2}$ isotropic Heisenberg $(X X X)$ chain is defined by the Hamiltonian

$$
\mathcal{H} \equiv J \sum_{i=1}^{L}\left[\frac{1}{2}\left(S_{i}^{+} S_{i+1}^{-}+S_{i}^{-} S_{i+1}^{+}\right)+S_{i}^{z} S_{i+1}^{z}\right],
$$

where $S_{i}^{ \pm} \equiv\left(\sigma_{i}^{x} \pm i \sigma_{i}^{y}\right) / 2$ are the ladder operators acting on the site $i$ of the chain, $S_{i}^{z} \equiv \sigma_{i}^{z} / 2$, and $\sigma_{i}^{x, y, z}$ the Pauli matrices. We set $J=1$ in (1) and use periodic boundary conditions identifying sites $L+1$ and 1 . Both the total spin
$S_{T}^{2} \equiv\left(\sum_{i} \vec{S}_{i}\right)^{2}$ and the total magnetization $S_{T}^{z} \equiv \sum_{i} S_{i}^{z}=$ $L / 2-M$, with $M$ being the number of down spins (particles), are conserved quantities for $\mathcal{H}$. Thus its eigenstates can be labeled by $S_{T}^{z}$ (equivalently by $M$, or the particle density $x \equiv M / L)$. Here we mainly consider the situation with fixed density $x=1 / 4$.

In the algebraic Bethe ansatz approach [100] the eigenstates of the $X X X$ chain in the sector with given $M$ are constructed from the so-called rapidities $\left\{\lambda_{\alpha}\right\}$ as $\left|\left\{\lambda_{\alpha}\right\}\right\rangle \equiv$ $\prod_{\beta=1}^{M} B\left(\lambda_{\beta}\right)|\Uparrow\rangle$. Here $|\Uparrow\rangle \equiv|\uparrow \uparrow \cdots \uparrow\rangle$ is the ferromagnetic ("vacuum") state, and $B(\lambda)$ a $2^{L} \times 2^{L}$ matrix [100]. The rapidities are obtained by solving the Bethe equations

$$
\arctan \left(\lambda_{\alpha}\right)=\frac{\pi}{L} J_{\alpha}+\frac{1}{L} \sum_{\beta \neq \alpha} \arctan \left(\frac{\lambda_{\alpha}-\lambda_{\beta}}{2}\right) .
$$

In principle, any choice of the so-called Bethe numbers $-L / 2<J_{\alpha} \leq L / 2\left(J_{\alpha} \in \frac{1}{2} \mathbb{Z}\right)$ identifies a set of solutions of (2), and an eigenstate of (1) thereof, with energy eigenvalue $E=\sum_{\alpha} 2 /\left(\lambda_{\alpha}^{2}+1\right)$. Although, in general, $\lambda_{\alpha} \in \mathbb{C}$, here we consider only real rapidities, i.e., $\lambda_{\alpha} \in \mathbb{R}, \forall \alpha$. Moreover, we focus on the eigenstates with maximum magnetization $S_{T}^{z}=S_{T}$, which implies $\lambda_{\alpha}<\infty, \forall \alpha$ [91]. The corresponding Bethe numbers are given as $-J_{\infty} \leq J_{\alpha} \leq J_{\infty}$, with $J_{\infty}=(L-1-M) / 2$ [91]. Finally, for each $L$ we consider $\sim 10^{4}$ eigenstates of (1), which are obtained by sampling uniformly the Bethe numbers $\left\{J_{\alpha}\right\}$. We should mention that here we choose $\Delta e \rightarrow \infty$, meaning that we are considering the infinite temperature canonical ensemble, instead of a microcanonical one. However, we anticipate that this does not affect our results.

Formally, for any eigenstate $\left|\left\{\lambda_{\alpha}\right\}\right\rangle$ one can write the cor- 

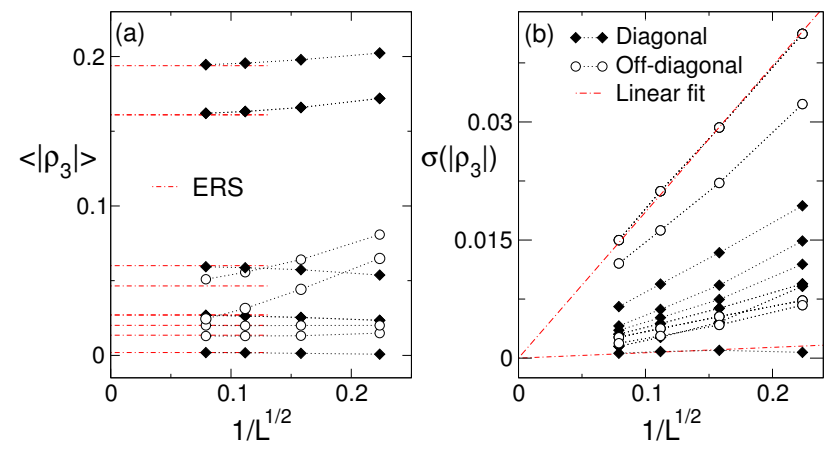

FIG. 2. Finite-size scaling of the eigenstate-to-eigenstate fluctuations of the three-spins reduced density matrix $\rho_{3}$ in the $X X X$ chain. (a) Eigenstate average $\left\langle\left|\rho_{3}\right|\right\rangle$ of $\rho_{3}$ plotted against $L^{-1 / 2}$ for chains with $L=20,40,80,160$. The average is over $\sim 10^{4}$ eigenstates in the sector with fixed particle density $x \equiv M / L=1 / 4, M$ being the number of down spins (particles). Full and empty symbols denote the diagonal and off-diagonal matrix elements of $\rho_{3}$, respectively. The dash-dotted lines denote $\rho_{3}^{E R S}$. (b) Fluctuations of $\left|\rho_{3}\right|$ : Standard deviation $\sigma\left(\left|\rho_{3}\right|\right)$ plotted versus $L^{-1 / 2}$. The dash-dotted lines are linear fits. The data suggest that $\sigma\left(\left|\rho_{3}\right|\right) \rightarrow 0$ in the thermodynamic limit.

responding reduced density matrix $\rho_{\ell}$ as $\left(\left\langle\left\{\lambda_{\alpha}\right\} \mid\left\{\lambda_{\alpha}\right\}\right\rangle=1\right)$

$$
\rho_{\ell}=\left\langle\left\{\lambda_{\alpha}\right\}\left|\prod_{j=1}^{\ell} \sigma_{j}^{\varepsilon_{j}}\right|\left\{\lambda_{\alpha}\right\}\right\rangle,
$$

where $\varepsilon_{j}=x, y, z, 0$, and $\sigma^{0} \equiv \mathbb{I}$ is the $2 \times 2$ identity matrix. Exact formulas for the multi-spin correlation functions appearing in (3) have been obtained recently within the algebraic Bethe ansatz approach [88, 89]. Their numerical implementation is a challenging task (cf. Ref. 87 for details), and the computational cost for calculating a generic matrix element of $\rho_{\ell}$ is roughly $\propto L^{\ell}$. Nonetheless, this method allows one to effectively calculate $\rho_{\ell}$ for $\ell \lesssim 6$ and chains with $L \sim 100$. We mention that this has been used in Ref. 87 to study entanglement properties of the excited states of the $X X Z$ chain.

\section{THE FLUCTUATIONS OF THE REDUCED DENSITY MATRIX}

Our main results are illustrated in Figure 1 focusing on $\rho_{2}$. Figure 1 (a) and (b) plot its diagonal elements $\rho_{2}[1,1]$ and $\rho_{2}[3,3]$ versus the eigenstate energy density $E / L$. We verified that $\rho_{2}[2,2]$ and $\rho_{2}[4,4]$ exhibit the same qualitative behavior. The only non-zero off-diagonal element $\rho_{2}[2,3]=\left(\rho_{2}[2,3]\right)^{*}$ is in general complex, and its real and imaginary parts are plotted in (c) and (d), respectively. In all panels the different symbols correspond to the chain lengths $L=20,40,160$ at fixed particle density $x=1 / 4$.

In the limit $L \rightarrow \infty$ the vast majority of the eigenstates exhibit the typical energy density $e_{t y p} \equiv E_{t y p} / L \approx$ $-0.2876 \cdots$ (cf. Figure 3 for a precise analysis). This suggests, as expected, that for large $L$ the infinite temperature canonical ensemble can be replaced with a microcanonical one centered around $e_{t y p}$. Correspondingly, Figure 1 (a)-(d) demonstrate that the eigenstate-to-eigenstate fluctuations of $\rho_{2}$ decrease upon increasing $L$, which implies that in the thermodynamic limit typical eigenstates yield the same $\rho_{2}$, and one has $\rho_{2} \rightarrow\left\langle\rho_{2}\right\rangle$. This allows us to conclude that the weak ETH holds for $\rho_{2}$. Finally, it is interesting to observe that, while in general $\rho_{2}[2,3] \in \mathbb{C}$, one has $\operatorname{Im}\left(\rho_{2}[2,3]\right) \approx 0$ at $L \rightarrow \infty$.

All these findings can be better characterized within the TBA approach by constructing an ensemble representative state (ERS) for the typical eigenstates. In the TBA, instead of the eigenstates, one considers the rapidity (particle) densities $\varrho_{p}(\lambda)$, whereas sums over eigenstates are replaced by a functional integral over $\varrho_{p}(\lambda)$ as [91]

$$
\left|\left\{\lambda_{\alpha}\right\}\right\rangle \rightarrow \varrho_{p}(\lambda), \quad \sum_{\left|\left\{\lambda_{\alpha}\right\}\right\rangle} \rightarrow \int \mathcal{D}\left[\varrho_{p}\right] e^{S_{Y Y}\left[\varrho_{p}\right]} .
$$

Here $\varrho_{h}$ denotes the "hole" (i.e., missing rapidities) density, and $S_{Y Y}\left[\varrho_{p}\right] \equiv L \int d \lambda\left[\left(\varrho_{p}+\varrho_{h}\right) \log \left(\varrho_{p}+\varrho_{h}\right)-\varrho_{p} \log \varrho_{p}-\right.$ $\left.\varrho_{h} \log \varrho_{h}\right]$ is the so-called Yang-Yang entropy. In the thermodynamic limit the integral in (4) is dominated by the saddle point $\bar{\varrho}_{p}$ of $S_{Y Y}\left[\varrho_{p}\right]$. At fixed density $x$, by imposing $\delta S_{Y Y} /\left.\delta \varrho_{p}\right|_{\varrho_{p}}=0$, one obtains that the ratio $\varrho_{h} / \varrho_{p}$ does not depend on $\lambda$, and it is given as $\bar{\varrho}_{h} / \bar{\varrho}_{p}=(1-2 x) / x$. Moreover, it is straightforward to derive from the Bethe equations that $2 \pi \bar{\varrho}_{p}(\lambda)=\int_{-\infty}^{+\infty} d \omega e^{-|\omega|+i \omega \lambda} /\left[(1-x) / x+e^{-2|\omega|}\right]$. The corresponding energy density, i.e., $e_{t y p}$, is readily obtained as $e_{t y p}=2 \int d \lambda \bar{\rho}_{p} /\left(1+\lambda^{2}\right)$ [91]. Notice that the peaking of the eigenstates energy density around $e_{t y p}$ (cf. Figure 1 (a)-(d) and Figure 3 (a)) in the thermodynamic limit is a consequence of $S_{Y Y}$ being extensive (cf. (4)).

For a finite chain, the condition $\bar{\varrho}_{h} / \bar{\varrho}_{p}=(1-2 x) / x$ implies that the saddle point is well approximated by the eigenstate (ERS) corresponding to the Bethe numbers $J_{\alpha}=$ $-(1-x) / 2 L+1 / 2+(1 / x-1) \alpha$, with $\alpha=0,1, \ldots, M-1$. Interestingly, this choice maximizes the "sparseness" of the Bethe numbers. The matrix elements of the resulting $\rho_{2}^{E R S}$ (for a chain with $L=80$ ) are reported in Figure 1 (a)-(d) as rhombi. For large chains one has $\rho_{2} \rightarrow \rho_{2}^{E R S}$ for typical eigenstates, which implies $\left\langle\rho_{2}\right\rangle \rightarrow \rho_{2}^{E R S}$, as shown in Figure 1 (i).

We now turn to discuss the fluctuations of $\rho_{2}$. These are illustrated in Figure 1 (ii), plotting the histograms of $\rho_{2}[3,3]$ for $L=40$ and $L=160$ (the latter is shifted vertically for visibility). Similar results are obtained for other matrix elements (not shown). The dashed line is a gaussian fit, suggesting that the fluctuations are normally distributed, as for non-integrable models [77]. Their amplitude vanishes upon increasing $L$, as expected (cf. Figure 1 (a)-(d)). This is numerically demonstrated for all matrix elements of $\rho_{2}$ in Figure 1 (iii), by plotting $\sigma\left(\rho_{2}\right)$ versus $L^{-1 / 2}$. Clearly, one has $\sigma\left(\rho_{2}\right) \propto L^{-1 / 2}$ (dash-dotted lines are linear fits).

One should remark that it is straightforward to derive that $\rho_{2}[1,1]=\left(1+4 G^{z z}\right) / 4$, with $G^{z z} \equiv\left\langle S_{i}^{z} S_{i+1}^{z}\right\rangle=$ $-\langle\mathcal{H} / L\rangle / 3$, where in the last step the $S U(2)$ invariance of $\mathcal{H}$ was used. This suggests that the weak ETH scenario for $\rho_{2}$ 


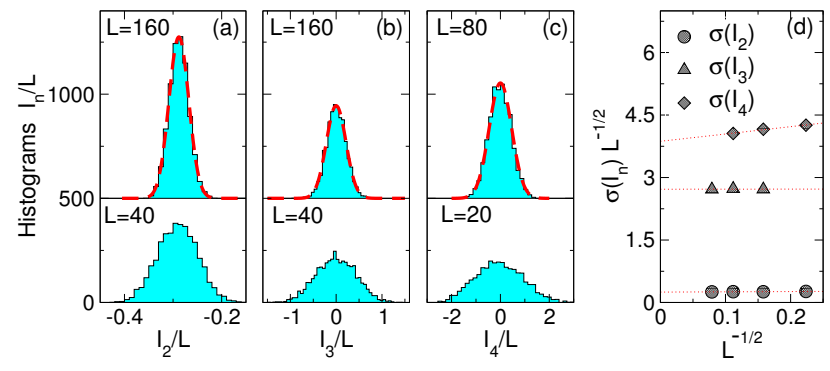

FIG. 3. Eigenstates expectation values (EEV) of the conserved charges $I_{n}(n=2,3,4)$ of the $X X X$ chain. (a)-(c) Histograms of $I_{n} / L$ for several chain lengths $L=20,40,80,160$. Here $I_{2}$ and $I_{3}$ are the energy $E$ and the energy current $J_{E}$, respectively. For each $L$ the data are obtained from $\sim 10^{4}$ eigenstates with fixed particle density $x \equiv M / L=1 / 4, M$ being the number of down spins. The histograms for the larger chains are shifted vertically for visibility. The dashed lines are gaussian fits. In the limit $L \rightarrow \infty$ one has $I_{2} / L \approx-0.3$, whereas $I_{3} / L \approx I_{4} / L \approx 0$. (d) Rescaled fluctuations $\sigma\left(I_{n}\right) L^{-1 / 2}$ plotted versus $L^{-1 / 2}$. The dotted lines are linear fits. Notice the finite extrapolations in the thermodynamic limit.

could be a trivial consequence of the behavior of the eigenstate energy density (cf. Figure 3 (a)). In order to provide a more stringent check of our result, in Figure 2 we discuss $\rho_{3}$. Figure 2 (a) plots all non-zero elements of $\left\langle\left|\rho_{3}\right|\right\rangle$ versus $L^{-1 / 2}$. Full and empty symbols denote diagonal and off-diagonal elements, respectively. The matrix elements of $\rho_{3}^{E R S}$ are shown as dash-dotted lines. Although finite-size effects are larger compared with $\rho_{2}$ (cf. Figure 1 (i)), one has $\left\langle\rho_{3}\right\rangle \rightarrow \rho_{3}^{E R S}$ at $L \rightarrow \infty$. The eigenstate-to-eigenstate fluctuations of $\rho_{3}$ are investigated in Figure 2 (b) plotting $\sigma\left(\left|\rho_{3}\right|\right)$ versus $L^{-1 / 2}$. Clearly, $\sigma\left(\left|\rho_{3}\right|\right) \propto L^{-1 / 2}$ is vanishing in the thermodynamic limit, as for $\rho_{2}$ (cf. Figure 1 (iii)). Finally, we should mention that similar qualitative behavior is observed for $\rho_{4}$.

\section{THE CONSERVED CHARGES}

It is interesting to consider the EEVs of the local conserved charges $I_{n}$ of the $X X X$ chain [94]. For each $n$, these are obtained as particular linear combinations of the matrix elements of $\rho_{n}$. Figure 3 plots the histograms of $I_{n} / L$ for $n=2,3,4$, (panels (a)-(c)) and $L=20,40,80,160$. Some of the histograms are shifted vertically for visibility. Here $I_{2} \equiv \mathcal{H}$, while $I_{3}$ is the energy current $I_{3}=J_{E} \equiv$ $\sum_{\alpha \beta \gamma} \epsilon_{\alpha \beta \gamma} \sigma_{i}^{\beta} \sigma_{i+1}^{\gamma} \sigma_{i+2}^{\alpha}$, with $\alpha, \beta, \gamma=x, y, z$ and $\epsilon_{\alpha \beta \gamma}$ the Levi-Civita symbol. The EEVs of $I_{n} / L$ are normally distributed around the typical values $\bar{I}_{n} / L \equiv\left\langle I_{n} / L\right\rangle$, as confirmed by the gaussian fits (dashed lines in the Figure). Interestingly, while $\bar{I}_{2} / L \approx-0.2876 \cdots$ (panel (a), see also Figure 1 ), one has $\bar{I}_{3} / L \approx \bar{I}_{4} / L \approx 0$ (panels (b) and (c)). The fluctuations of $I_{n} / L$ decay as $L^{-1 / 2}$ in the thermodynamic limit, reflecting the behavior of $\rho_{\ell}$ (cf. Figure 1 and 2). This is verified in Figure $3(\mathrm{~d})$ plotting $\sigma\left(I_{n}\right) L^{-1 / 2}$ versus $L^{-1 / 2}$. Clearly, $\sigma\left(I_{n}\right) L^{-1 / 2} \rightarrow$ const at $L \rightarrow \infty$ (the dotted lines are linear fits). Physically, the behavior of $I_{2}$ reflects the specific heat per site, $C_{v}=\beta^{2} / L\left(\left\langle I_{2}^{2}\right\rangle-\left\langle I_{2}\right\rangle^{2}\right)$, being finite in the
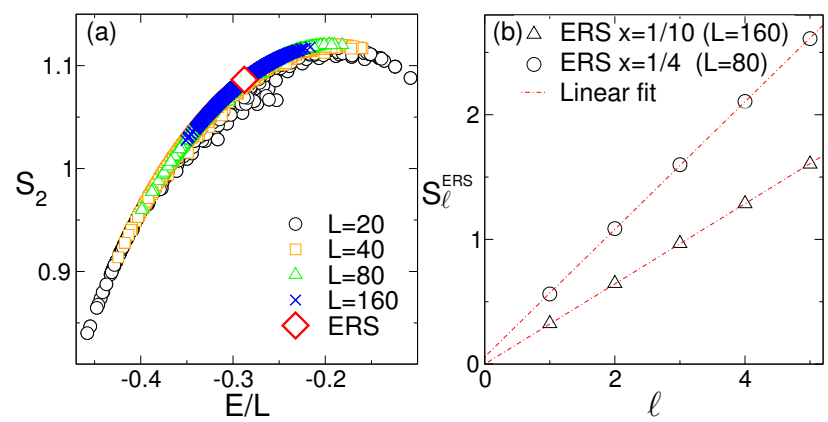

FIG. 4. Entanglement entropy of the eigenstates of the spin- $\frac{1}{2}$ $X X X$ chain. (a) Two-spin von Neumann entropy $S_{2}$ plotted versus the eigenstates energy density $E / L$. Different symbols correspond to chain lengths $L=20,40,80,160$. The rhombus denotes $S_{2}^{E R S}$. (b) $S_{\ell}^{E R S}$ as a function of $\ell=1, \ldots, 5$, and for $L=80,160$. Circles and triangles correspond to particle densities $x \equiv M / L=1 / 4,1 / 10$, respectively. Here $M$ is the number of down spins (particles). The dashed-dotted lines are linear fits. For both $x=1 / 4,1 / 10$ the volume law $S_{\ell}^{E R S} \propto \ell$ is clearly visible.

thermodynamic limit. Likewise, the result for $I_{3}$ is related to the finite Drude weight for the energy current [101-103].

\section{ENTANGLEMENT PROPERTIES}

We now turn to discuss how the scenario outlined so far is reflected in the entanglement entropy $S_{\ell}$ of the eigenstates of the $X X X$ spin chain. Notice that entanglement properties of excited states of many-body Hamiltonians have attracted increasing attention recently [87, 104-117]. Figure 4 (a) plots the two-spin entropy $S_{2}$ versus $E / L$ for different eigenstates of the $X X X$ chain. The eigenstate-to-eigenstate fluctuations of $S_{2}$ decrease upon increasing $L$, reflecting the behavior of $\rho_{2}$ (cf. Figure 1). In particular, in the thermodynamic limit one has $S_{2} \rightarrow S_{2}^{E R S}$. The scaling behavior of $S_{\ell}^{E R S}$, as a function of $\ell$ is investigated in Figure 4 (b) plotting $S_{\ell}^{E R S}$ versus $1 \leq \ell \leq 5$ for chains of length $L=80,160$. We restrict ourselves to density $x=1 / 4$ (high density) and $x=1 / 10$ (low density). In both cases Figure 4 (b) provides a robust evidence of the volume law $S_{\ell}^{E R S} \sim \ell$ (the dash-dotted lines are linear fits). This allows us to conclude that typical midspectrum eigenstates of the $X X X$ chain exhibit extensive entanglement (see also Ref. 118), whereas other behaviors (for instance a logarithmic one as $\propto \log (\ell)$ ) are associated with rare eigenstates. Notice that this should be reflected in the extensive behavior of the entanglement entropy after a quantum quench [41, 119-122].

\section{CONCLUSIONS}

We performed a careful finite-size scaling analysis of the eigenstate thermalization hypothesis (ETH) in the spin- $\frac{1}{2}$ isotropic Heisenberg $(X X X)$ chain. Precisely, we focused on the $\ell$-spin reduced density matrix $\rho_{\ell}$. Using a numerical implementation [87] of state-of-the-art algebraic Bethe ansatz 
results $[88,89]$, we provided numerical evidence that ETH holds for typical eigenstates (weak ETH scenario). Specifically, we numerically demonstrated that the eigenstate-toeigenstate fluctuations of $\rho_{\ell}$ decay as $L^{-1 / 2}$ in the thermodynamic limit. This is in contrast with the exponential decay that is observed in generic non-integrable models [77], for which the strong ETH holds. Although here we considered one specific model, and we restricted ourselves to $\rho_{\ell}$, it is natural to expect that the same result holds in other integrable spin systems and for generic local observables. Finally, we numerically verified that typical mid-spectrum eigenstates of the $X X X$ chain exhibit extensive entanglement entropy.

Our results open several new possible research avenues. For instance, while here we restricted ourselves to eigenstates obtained from real solutions of the Bethe equations, it would be interesting to generalize our results including complex solutions (strings) [91]. Notice that for $\ell=2$ this should be straightforward using the techniques developed in Ref. 123125. Furthermore, it would be useful to characterize the scal- ing behavior of the entanglement entropy in eigenstates away from the middle of the energy spectrum. An intriguing direction would be to investigate the relationship between entanglement and the spectrum of the conserved charges (other than the Hamiltonian). In particular, it would be enlightening to study whether eigenstates at the edges of the spectrum give non-extensive entanglement entropy. Finally, it would interesting to extend our analysis to off-diagonal matrix elements of local observables, which are important to understand the approach to relaxation to a steady state after quantum quenches $[81,126]$.

Acknowledgments. - I would like to thank P. Calabrese, F. H. L. Essler, M. Fagotti, F. Heidrich-Meisner, A. M. Läuchli, and A. Lazarides for enlightening discussions and comments. In particular, I would like to thank F. H. L. Essler for many useful insights during the completion of this work and P. Calabrese and M. Fagotti for useful comments about the manuscript. I acknowledge financial support by the ERC under Starting Grant 279391 EDEQS.
[1] J. Gemmer, M. Michel, and G. Mahler, Lect. Not. Phys. 657 (2004).

[2] M. Rigol, V. Dunjko, V. Yurovsky, and M. Olshanii, Phys. Rev. Lett. 98, 050405 (2007).

[3] S. Popescu, A. J. Short, and A. Winter, Nature Physics 2, 754 (2006).

[4] M. Rigol, V. Dunjko, and M. Olshanii, Nature 452, 854 (2008).

[5] A. Polkovnikov, K. Sengupta, and M. Vengalattore, Rev. Mod. Phys. 83, 863 (2011).

[6] V. I. Yukalov, Laser Phys. Lett. 7, 485 (2011).

[7] J. Eisert., M. Friesdorf, and C. Gogolin, arXiv:1408.5148.

[8] C. Kollath, A. M. Läuchli, and E. Altman, Phys. Rev. Lett. 98, 180601 (2007).

[9] S. R. Manmana, S. Wessel, R. M. Noack, and A. Muramatsu, Phys. Rev. Lett. 98, 210405 (2007).

[10] P. Calabrese and J. Cardy, J. Stat. Mech. P06008 (2007).

[11] M. Cramer, C. M. Dawson, J. Eisert, and T. J. Osborne, Phys. Rev. Lett. 100, 030602 (2008).

[12] T. Barthel and U. Schollwöck, Phys. Rev. Lett. 100, 100601 (2008).

[13] M. Cramer, A. Flesch, I. P. McCulloch, U. Schollwöck, and J. Eisert, Phys. Rev. Lett. 101, 063001 (2008).

[14] M. Kollar and M. Eckstein, Phys. Rev. A 78, 013626 (2008).

[15] A. Iucci and M. A. Cazalilla, Phys. Rev. A 80, 063619 (2009).

[16] S. Sotiriadis, P. Calabrese, and J. Cardy, EPL 87, 20002 (2009).

[17] G. Roux, Phys. Rev. A 79, 021608 (2009).

[18] M. Rigol, Phys. Rev. Lett. 103, 100403 (2009).

[19] M. Rigol, Phys. Rev. A 80, 053607 (2009).

[20] P. Barmettler, M. Punk, V. Gritsev, E. Demler, and E. Altman, Phys. Rev. Lett. 102, 130603 (2009).

[21] P. Barmettler, M. Punk, V. Gritsev, E. Demler, and E. Altman, New J. Phys. 12, 055017 (2010).

[22] M. Cramer and J. Eisert, New J. Phys. 12, 055020 (2010).

[23] A. Flesch, M. Cramer, I. P. McCulloch, U. Schollwöck, and J. Eisert, Phys. Rev. A 78, 033608 (2008).

[24] G. Roux, Phys. Rev. A 81, 053604 (2010).

[25] D. Fioretto and G. Mussardo, New J. Phys. 12, 055015 (2010).
[26] G. Biroli, C. Kollath, and A. M. Läuchli, Phys. Rev. Lett. 105, 250401 (2010).

[27] L. F. Santos and M. Rigol, Phys. Rev. E 82, 031130 (2010).

[28] M. C. Bañuls, J. I. Cirac, and M. B. Hastings, Phys. Rev. Lett. 106, 050405 (2011).

[29] P. Calabrese, F. H. L. Essler, and M. Fagotti, Phys. Rev. Lett. 106, 227203 (2011).

[30] C. Gogolin, M. P. Mueller, and J. Eisert, Phys. Rev. Lett. 106, 040401 (2011).

[31] M. Rigol and M. Fitzpatrick, Phys. Rev. A 84, 033640 (2011).

[32] T. Caneva, E. Canovi, D. Rossini, G. E. Santoro, and A. Silva, J. Stat. Mech. (2011) P07015.

[33] L. Santos, A. Polkovnikov, and M. Rigol, Phys. Rev. Lett. 107, 040601 (2011).

[34] A. C. Cassidy, C. W. Clark, and M. Rigol, Phys. Rev. Lett. 106, 140405 (2011).

[35] F. H. L. Essler, S. Evangelisti, and M. Fagotti, Phys. Rev. Lett. 109, 247206 (2012)

[36] M. A. Cazalilla, A. Iucci, and M.-C. Chung, Phys. Rev. E 85, 011133 (2012).

[37] J. Mossel and J.-S. Caux, New J. Phys. 14075006 (2012).

[38] M. Rigol and M. Srednicki, Phys. Rev. Lett. 108, 110601 (2012).

[39] J. Mossel and J.-S. Caux, J. Phys. A: Math. Theor. 45, 255001 (2012).

[40] M. Fagotti and F. H. L. Essler, Phys. Rev. B 87, 245107 (2013).

[41] M. Fagotti, Phys. Rev. B 87, 165106 (2013).

[42] M. Collura, S. Sotiriadis, and P. Calabrese, Phys. Rev. Lett. 110, 245301 (2013).

[43] J.-S. Caux and F. H. L. Essler, Phys. Rev. Lett. 110, 257203 (2013).

[44] G. Mussardo, Phys. Rev. Lett. 111, 100401 (2013).

[45] M. Kormos, A. Shashi, Y.-Z. Chou, J.-S. Caux, and A. Imambekov, Phys. Rev. B 88, 205131 (2013).

[46] B. Bertini, D. Schuricht, and F. H. L. Essler, arXiv:1405.4813 (2014).

[47] S. Sotiriadis and P. Calabrese, J. Stat. Mech. (2014) P07024.

[48] F. H. L. Essler, S. Kehrein, S. R. Manmana, and N. J. Robinson, Phys. Rev. B 89, 165104 (2014). 
[49] M. Fagotti, M. Collura, F. H. L. Essler, and P. Calabrese, Phys. Rev. B 89, 125101 (2014).

[50] M. Fagotti, J. Stat. Mech. (2014) P03016.

[51] B. Wouters, J. De Nardis, M. Brockmann, D. Fioretto, M. Rigol, and J.-S. Caux, Phys. Rev. Lett. 113, 117202 (2014).

[52] B. Pozsgay, M. Mestyán, M. A. Werner, M. Kormos, G. Zarànd, and G. Takács, Phys. Rev. Lett. 113, 117203 (2014).

[53] M. Greiner, O. Mandel, T. Hänsch, and I. Bloch, Nature (London) 419, 51 (2002).

[54] T. Kinoshita, T. Wenger, and D. S. Weiss, Nature (London) 440, 900 (2008).

[55] S. Hofferberth, I. Lesanovsky, B. Fischer, T. Schumm, and J. Schiedmayer, Nature (London) 449, 324 (2007).

[56] I. Bloch, J. Dalibard, and W. Zwerger, Rev. Mod. Phys. 80, 885 (2008)

[57] S. Trotzky, Y.-A. Chen, A. Flesch, I. P. McCulloch, U. Schollwöck, J. Eisert, and I. Bloch, Nature Phys. 8, 325 (2012).

[58] M. Gring, M. Kuhnert, T. Langen, T. Kitagawa, B. Rauer, M. Schreitl, I. Mazets, D. A. Smith, E. Demler, and J. Schmiedmayer, Science 337, 6100 (2012).

[59] M. Cheneau, P. Barmettler, D. Poletti, M. Endres, P. Schaua, T. Fukuhara, C. Gross, I. Bloch, C. Kollath, and S. Kuhr, Nature (London) 481, 484 (2012).

[60] U. Schneider, L. Hackeruller, J. P. Ronzheimer, S. Will, S. Braun, T. Best, I. Bloch, E. Demler, S. Mandt, D. Rasch, and A. Rosch, Nature Phys. 8, 213 (2012).

[61] M. Kuhnert, R. Geiger, T. Langen, M. Gring, B. Rauer, T. Kitagawa, E. Demler, D. Adu Smith, and J. Schmiedmayer, Phys. Rev. Lett. 110, 090405 (2013).

[62] T. Langen, R. Geiger, M. Kuhnert, B. Rauer, and J. Schmiedmayer, Nature Phys. 9, 640 (2013).

[63] F. Meinert, M. J. Mark, E. Kirilov, K. Lauber, P. Weinmann, A. J. Daley, and H.-C. Nagerl, Phys. Rev. Lett. 111, 053003 (2013).

[64] T. Fukuhara, A. Kantian, M. Endres, M. Cheneau, P. Schaua, S. Hild, C. Gross, U. Schollwöck, T. Giamarchi, I. Bloch, and S. Kuhr, Nature Phys. 9, 235 (2013).

[65] J. P. Ronzheimer, M. Schreiber, S. Braun, S. S. Hodgman, S. Langer, I. P. McCulloch, F. Heidrich-Meisner, I. Bloch, and U. Schneider, Phys. Rev. Lett. 110, 205301 (2013).

[66] S. Braun, M. Friesdorf, S. Hodgman, M. Schreiber, J. Ronzheimer, A. Riera, M. del Rey, I. Bloch, J. Eisert, and U. Schneider, arXiv:1403.7199.

[67] J. M. Deutsch, Phys. Rev. A 43, 2046 (1991).

[68] M. Srednicki, Phys. Rev. E 50, 888 (1994).

[69] M. Srednicki, J. Phys. A 29, L75 (1996).

[70] M. Srednicki, J. Phys. A 32, 1163 (1999).

[71] S. Goldstein, J. L. Lebowitz, R. Tumulka, and N. Zanghí, Phys. Rev. Lett. 96, 050403 (2006).

[72] S. Goldstein, J. L. Lebowitz, C. Mastrodonato, R. Tumulka, and N. Zanghi, Proc. R. Soc. A 466, 3203 (2010).

[73] S. Goldstein, J. L. Lebowitz, R. Tumulka, and N. Zanghi, Eur. Phys. J. H 35, 173 (2010).

[74] T. N. Ikeda, Y. Watanabe, and M. Ueda, Phys. Rev. E 84, 021130 (2011).

[75] T. N. Ikeda, Y. Watanabe, and M. Ueda, Phys. Rev. E 87, 012125 (2013).

[76] R. Steinigeweg, J. Herbrych, and P. Prelovšek, Phys. Rev. E 87, 012118 (2013).

[77] W. Beugeling, R. Moessner, and M. Haque, Phys. Rev. E 89, 042112 (2014).
[78] P. R. Zangara, A. D. Dante, E. J. Torres-Herrera, H. M. Pastawski, A. Iucci, and L. Santos, Phys. Rev. E 88, 032913 (2013).

[79] R. Steinigeweg, A. Khodja, H. Niemeyer, C. Gogolin, and J. Gemmer, Phys. Rev. Lett. 112, 130403 (2014).

[80] S. Sorg, L. Vidmar, L. Pollet, and F. Heidrich-Meisner, arXiv: $1405.5404 \mathrm{v} 2$.

[81] W. Beugeling, R. Moessner, and M. Haque, arXiv:1407.2043.

[82] V. Khemani, A. Chandran, H. Kim, and S. L. Sondhi, arXiv: 1406.4863.

[83] H. Kim, T. N. Ikeda, and D. Huse, arXiv:1408.0535.

[84] L. Bonnes, F. H. L. Essler, and A. M. Läuchli, arXiv: 1404.4062 (2014).

[85] G. B. Brandino, A. de Luca, R. M. Konik, and G. Mussardo, Phys. Rev. B 85, 214435 (2012).

[86] J.-S. Caux and J. Mossel, J. Stat. Mech. (2011) P02023.

[87] V. Alba, M. Fagotti, and P. Calabrese, J. Stat. Mech. (2009) P10020.

[88] N. Kitanine, J. M. Maillet, and V. Terras, Nucl. Phys. B 554, 647 (1999).

[89] N. Kitanine, J. M. Maillet, and V. Terras, Nucl. Phys. B 567, 554 (2000).

[90] L. Amico, R. Fazio, A. Osterloh, and V. Vedral, Rev. Mod. Phys. 80, 517 (2008).

[91] M. Takahashi, Thermodynamics of one-dimensional solvable models, Cambridge University Press 1999.

[92] C. N. Yang and C. P. Yang, J. Math. Phys. 10, 1115 (1969).

[93] M. Takahashi, Prog. Theor. Phys. 46, 401 (1971).

[94] M. P. Grabowski and P. Mathieu, Ann. Phys. N.Y. 243, 299 (1995).

[95] L. F. Santos, F. Borgonovi, and F. M. Izrallev, Phys. Rev. Lett. 108094102 (2012).

[96] L. F. Santos, F. Borgonovi, and F. M. Izrallev, Phys. Rev. E 85 036209 (2012).

[97] J. Eisert, M. Cramer, and M. B. Plenio, Rev. Mod. Phys. 82, 277 (2009).

[98] P. Calabrese, J. Cardy, and B. Doyon Eds., Special issue: Entanglement entropy in extended systems, J. Phys. A 42, 50 (2009).

[99] P. Calabrese and J. Cardy, J. Phys. A 42504005 (2009).

[100] V. E. Korepin, N. M. Bogoliubov, and A. G. Izergin, Quantum Inverse Scattering Methods and Correlation Functions, Cambridge University Press 1997.

[101] X. Zotos and P. Prelovšek, Phys. Rev. B 53, 983 (1996).

[102] H. Castella and X. Zotos, Phys. Rev. B 54, 4375 (1996).

[103] X. Zotos, F. Naef, and P. Prelovšek, Phys. Rev. B 55, 11029 (1997)

[104] F. C. Alcaraz, M. I. Berganza, and G. Sierra, Phys. Rev. Lett. 106, 201601 (2011).

[105] I. Pizorn, arXiv:1202.3336.

[106] M. I. Berganza, F. C. Alcaraz, and G. Sierra, J. Stat. Mech. (2012) P01016.

[107] G. Wong, I. Klich, L. A. P. Zayas, and D. Vaman, JHEP 12 (2013) 020

[108] M. Storms, and R. R. P. Singh, Phys. Rev. E 89, 012125 (2014).

[109] R. Berkovits, Phys. Rev. B 87, 075141 (2013).

[110] F. H. L. Essler, A. M. Läuchli, and P. Calabrese, Phys. Rev. Lett. 110, 115701 (2013).

[111] M. Nozaki, T. Numasawa, T. Takayanagi, Phys. Rev. Lett. 112, 111602 (2014).

[112] G. Ramirez, J. Rodriguez-Laguna, and G. Sierra, arXiv:1402.5015. 
[113] F. Ares, J. G. Esteve, F. Falceto, and E. Sánchez-Burillo, arXiv:1401.5922.

[114] Y. Huang, and J. Moore, arXiv:1405.1817.

[115] T. Pálmai, arXiv:1406.3182.

[116] J. Mölter, T. Barthel,U. Schollwöck, and V. Alba, arXiv:1407.0066.

[117] H.-H. Lai and K. Yang, arXiv:1409:1224

[118] J. Sato, B. Aufgebauer, H. Boos, F. Göhmann, A. Klümper, M. Takahashi, and C. Trippe, Phys. Rev. Lett. 106, 257201 (2011).

[119] M. Fagotti and P. Calabrese, Phys. Rev. A 78, 010306 (2008).

[120] V. Gurarie, J. Stat. Mech. (2014) P02014.
[121] M. Collura, M. Kormos, and P. Calabrese, J. Stat. Mech. (2014) P01009.

[122] M. Kormos, L. Bucciantini, and P. Calabrese, EPL 107, 40002 (2014).

[123] J.-S. Caux and J.-M. Maillet, Phys. Rev. Lett. 95, 077201 (2005).

[124] J.-S. Caux, R. Hagemans and J.-M. Maillet, J. Stat. Mech. P09003 (2005).

[125] J.-S. Caux, J. Math. Phys. 50, 095214 (2009).

[126] E. Khatami, G. Pupillo, M. Srednicki, and M. Rigol, Phys. Rev. Lett. 111, 050403 (2013). 